\title{
3010 Induction of a Guiding Sheath to Carotid Technical Artery without Intra-aortic Manipulation by Using a Pigtail-type Inner Catheter: Technical Note and Report of Two Cases
}

Shin Hirota, Masataka Yoshimura, Shoko Fujii, Juri Kiyokawa, and Shinji Yamamoto

Objective: Catheter operations in the aortic arch with atheromatous ulcers, aortic aneurysms, or artificial blood vessels increase the risk of complications, such as cholesterin embolism. We report two cases in which a guiding sheath was directly guided into the right carotid artery through the radial artery using a $5 \mathrm{Fr} 110-\mathrm{cm}$ pigtail catheter as an inner catheter to avoid the above complications.

Case Presentations: Case 1: A 51-year-old female with an anterior communicating artery aneurysm. Balloon-assisted embolization of the aneurysm was performed using this method as she had undergone aortic arch replacement.

Case 2: A 74-year-old male with stenosis of the right internal carotid artery. Carotid artery stenting (CAS) was conducted using this method due to shaggy aorta.

Conclusion: This method may be simple and useful for avoiding the complexity of catheter exchange and risk of intraaortic-arch manipulation.

Keywords > pigtail catheter, transradial, shaggy aorta, carotid artery stenting, coil embolization

\section{Introduction}

There are three approaches for neuroendovascular treatment: transfemoral, -radial, and -brachial approaches (TFA, TRA, and TBA, respectively). A merit of TRA and TBA is that these two do not involve descending aorta and more distal vessels. However, in many cases, approaches involving the aortic arch are necessary, and the presence of lesions, such as atheromatous ulcers, aortic aneurysms, or previously operated vessels may increase the risk of complications, including distal/cholesterin embolism and so

$\overline{\text { Department of Neurosurgery, Tsuchiura Kyodo General Hospital, }}$ Tsuchiura, Ibaraki, Japan

Received: May 8, 2017; Accepted: October 8, 2017

Corresponding author: Shin Hirota. Department of Neurosurgery, Tsuchiura Kyodo General Hospital, 4-1-1 Otsuno, Tsuchiura, Ibaraki 300-0028, Japan

Email: hiroshin_21@yahoo.co.jp

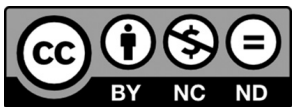

This work is licensed under a Creative Commons Attribution-NonCommercialNoDerivatives International License.

(C)2018 The Japanese Society for Neuroendovascular Therapy forth. ${ }^{1-3)}$ Misaki et al. ${ }^{4)}$ have established a method to induce a 5 Fr guiding sheath into the carotid artery without passing the aorta by using a pigtail catheter. At first, they induced a pigtail catheter into the brachiocephalic trunk via TBA and a guidewire through the catheter into the carotid artery. Subsequently, they exchanged catheters twice to finally induce a $5 \mathrm{Fr}$ guiding sheath. In this study, we report two patients in whom a $6 \mathrm{Fr}$ guiding sheath could be guided into the right carotid artery through the radial artery using an OptiFlush 5 Fr 110-cm pigtail catheter (Terumo Corporation, Tokyo, Japan) as an inner catheter without catheter exchanges.

\section{Case Presentations}

\section{Case 1}

Case: A 51-year-old female.

Medical history: Surgically treated aortic arch aneurysm and hypertension.

Present illness: An anterior communicating artery aneurysm measuring $5 \mathrm{~mm}$ was detected on preoperative examination of an ascending aortic arch aneurysm. The patient 


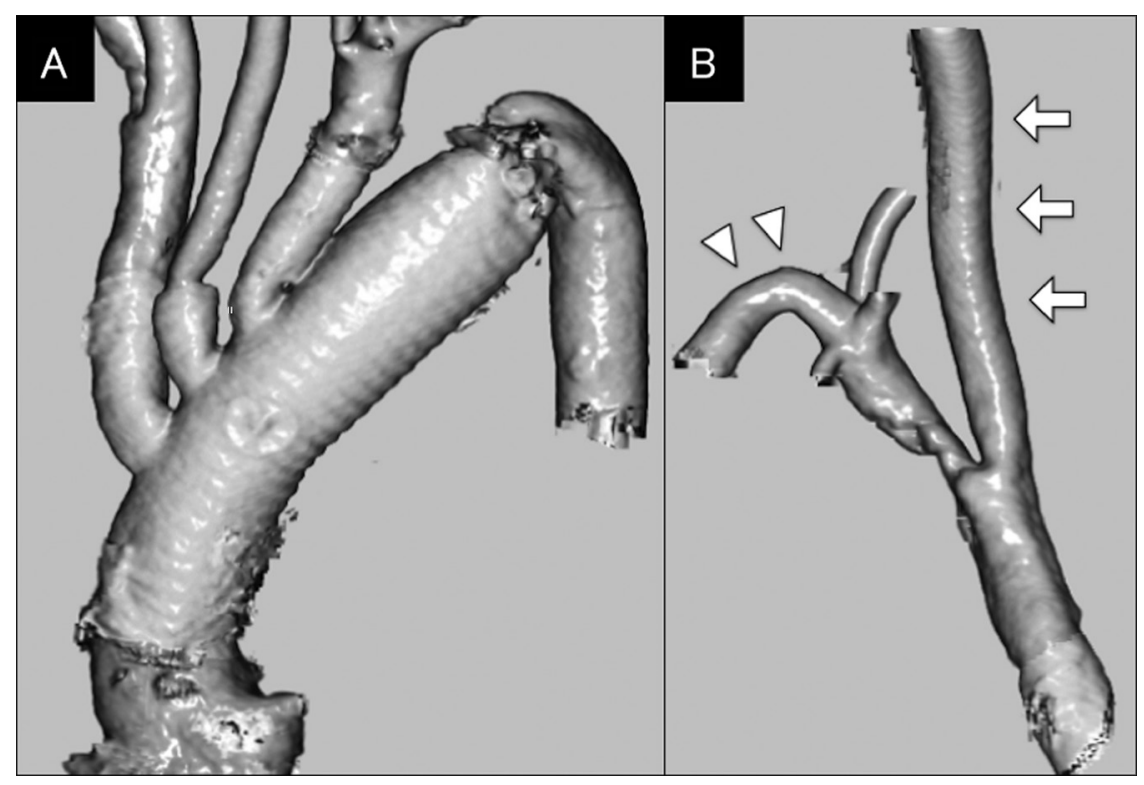

Fig. 1 Three-dimensional CTA in case 1. (A) Left oblique view of the thoracic aorta. The aortic arch and its branches are replaced with the vascular graft. (B) Antero-posterior view of the right subclavian artery (arrowheads) and the right common carotid artery (arrows), showing a sharp hairpin-curve between the two.

wished to undergo neuroendovascular treatment, and was referred to our department 6 months after the aortic arch replacement. Regarding the approach route to the anterior communicating aneurysm through dominant right $\mathrm{A} 1$, the replaced aortic arch could be risk factors. In addition, the anastomotic site between the artificial blood vessel and descending aorta was flexed at 45 degrees (Fig. 1A), which made us perform the endovascular treatment via right TRA. Since intra-artificial graft manipulation should be avoided, and the right subclavian and common carotid arteries were making a very sharp angle (25 degrees) (Fig. 1B), we chose a pigtail catheter to induce a guidewire directly into the right common carotid artery through the right subclavian artery, as reported by Mistake et al. ${ }^{4)}$

Endovascular treatment: Under general anesthesia, neuroendovascular treatment was performed. A 6 Fr 90-cm guiding sheath was guided into the subclavian artery via right TRA. To avoid the complexity of catheter exchange, a $5 \mathrm{Fr}$ $110-\mathrm{cm}$ pigtail catheter, which is long enough to guide the 6 Fr 90-cm guiding sheath, was selected as an inner catheter. The tip of the pigtail catheter was guided into the brachiocephalic trunk (Fig. 2A). The pigtail shape became J-shaped just after insertion of a guidewire, which made the guidewire insertion into the common carotid artery quite easy (Fig. 2B). The guidewire was slightly pulled, and the pigtail catheter tip was transferred into the common carotid artery origin. The guidewire was safely induced into the internal carotid artery afterward (Fig. 2C). By fixing both the pigtail catheter and the guidewire to the position as a guiding axis, the guiding sheath was inserted into the common carotid artery (Fig. 2D). It took 4 minutes from pigtail-catheter insertion until the guiding-sheath insertion into carotid artery. The inner catheter was changed, and the guiding sheath was inserted into the high-level internal carotid artery. Balloon-assisted coil embolization was performed. The guiding sheath was removed without reversing heparin, and compressive hemostasis with a BLEED SAFE (Medikit Co., Ltd., Tokyo, Japan) was conducted.

Postoperative course: She was discharged without complications, such as deficits, radial artery occlusion, or ischemic symptoms of the upper limbs, or appearance of new lesions on MRI (Fig. 3). The patient was discharged.

\section{Case 2}

Case: A 74-year-old male.

Medical history: Hypertension, chronic obstructive pulmonary disease, and reflux esophagitis.

Present illness: He was diagnosed with the right internal carotid artery stenosis (North American Symptomatic Carotid Endarterectomy Trial [NASCET]: 60\%) at a local clinic, and referred to our department. 3D CTA showed marked calcification and ulcer formation of the aortic arch (Fig. 4A and 4B). In addition, the route from the right 


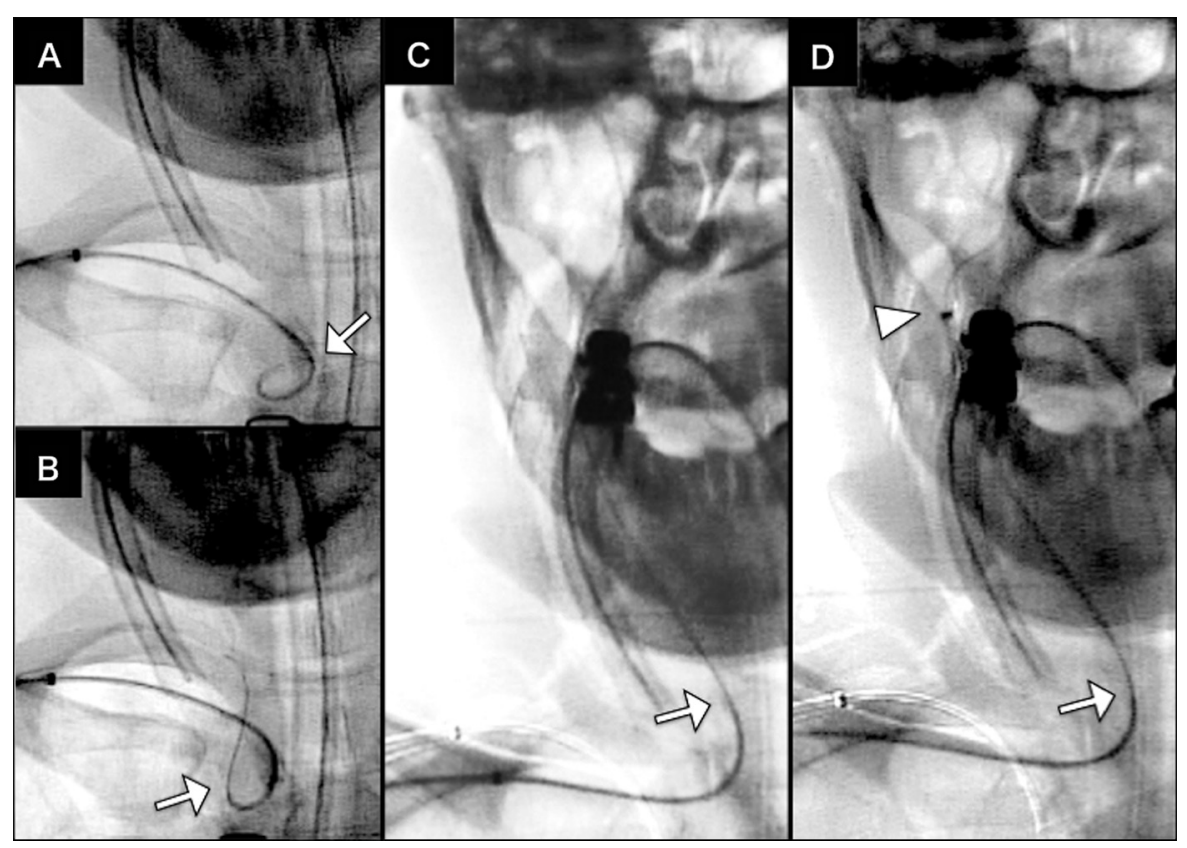

Fig. 2 Fluoroscopy during the endovascular treatment in case 1. (A) The $6 \mathrm{Fr}$ guiding sheath is placed in the right subclavian artery, and the $5 \mathrm{Fr}$ pigtail catheter (arrow) is placed in the innominate artery. (B) The guidewire unrolls the loop of the pigtail catheter and advances directly into the right common carotid artery. The arrow shows the tip of the pigtail catheter in the innominate artery. (C) The tip of the pigtail catheter (arrow) is inserted into the right common carotid artery, and the guidewire is further advanced into the right internal carotid artery. (D) The $6 \mathrm{Fr}$ guiding sheath (arrowhead) is successfully placed in the right common carotid artery. The tip of the pigtail catheter (arrow) is still placed in the right common carotid artery.

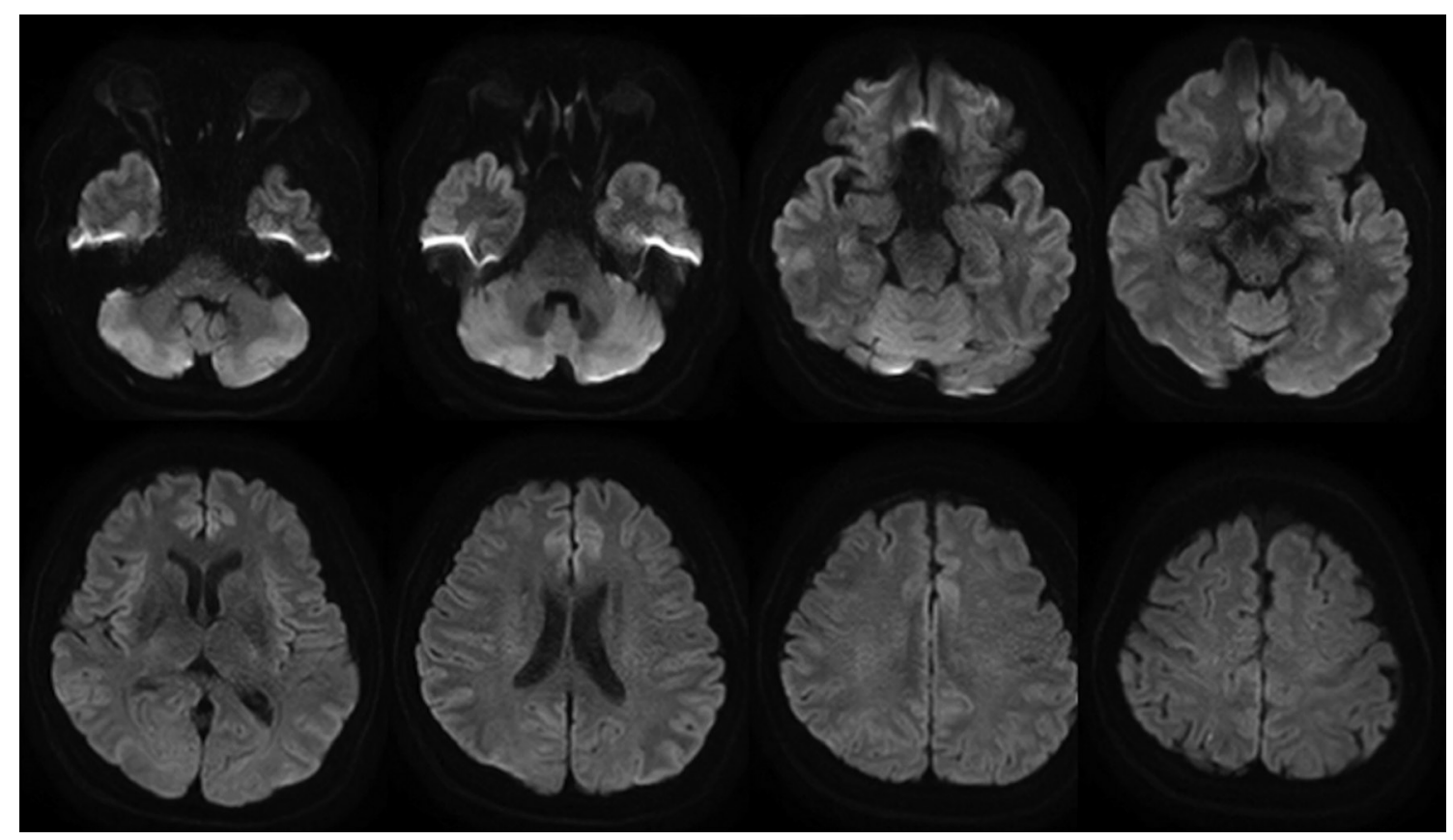

Fig. 3 Postoperative MRI in case 1, showing no high-signal intensities in diffusion-weighted images. 


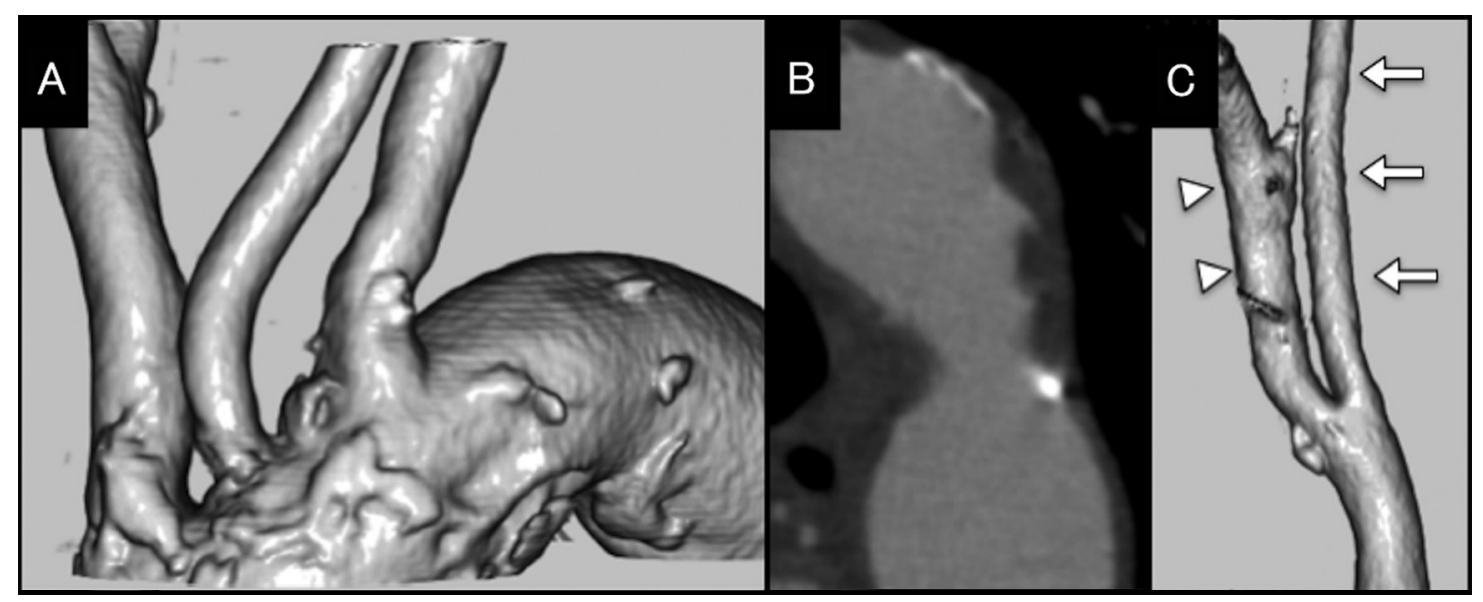

Fig. 4 Three-dimensional CTA in case 2. (A) Left oblique view of the aortic arch showing calcified plaques and irregular dimples on the aortic wall. (B) Axial section of the aortic arch, showing thickened and shaggy wall of the aorta. (C) Right lateral view of the right subclavian artery (arrowheads) and the right common carotid artery (arrows), showing a sharp hairpin-curve between the two.

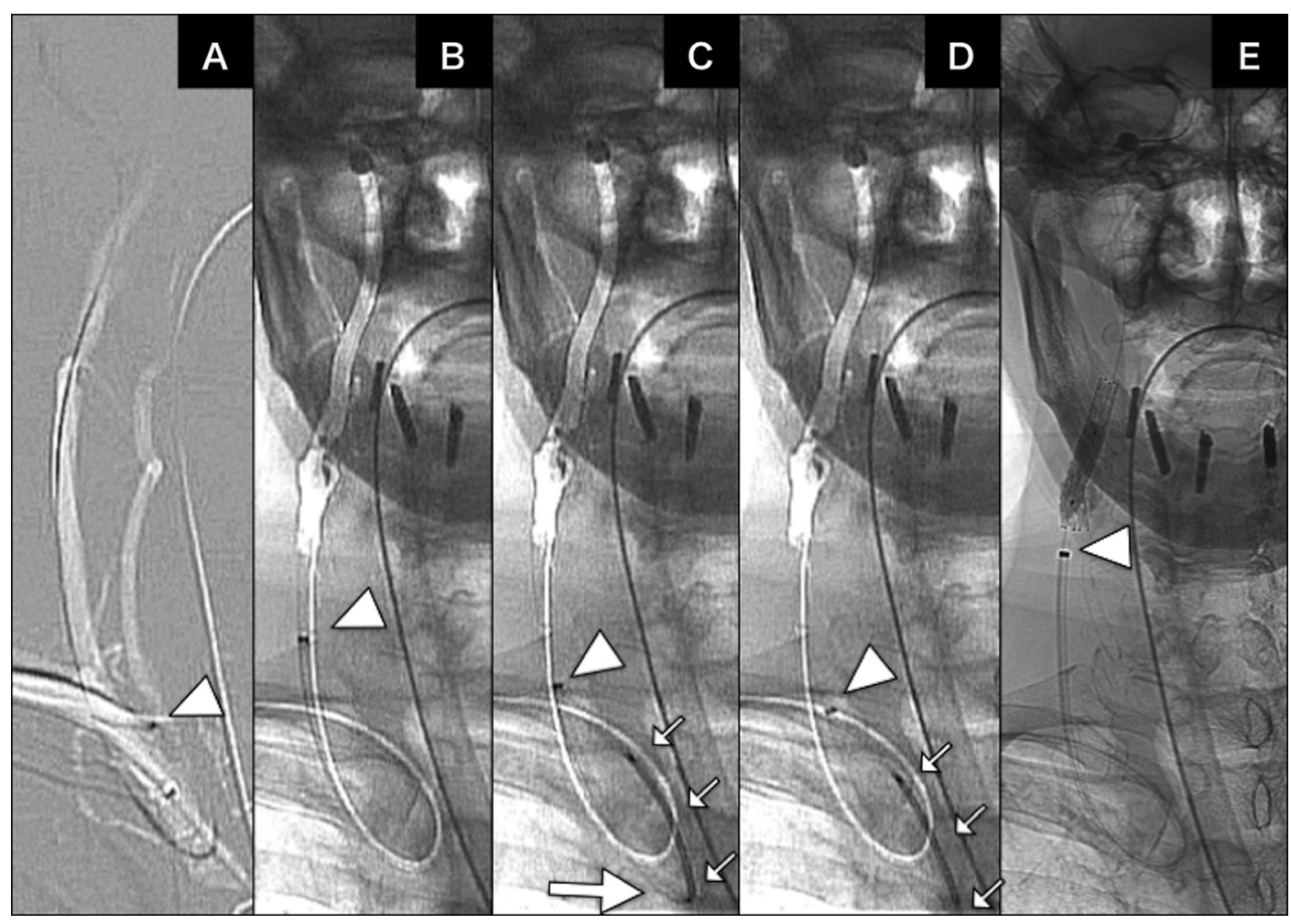

Fig. 5 Fluoroscopy during the endovascular treatment in case 2. (A) The 6 Fr guiding sheath is placed in the right subclavian artery (arrowhead), and the guidewire advances directly into the right external carotid artery. (B) The $6 \mathrm{Fr}$ guiding sheath (arrowhead) is successfully placed in the right common carotid artery. The distal protection balloon is inflated in the distal internal carotid artery. (C and $\mathbf{D}$ ) The guiding sheath (arrowhead) is plugged up by the stent-delivery-system (small arrows) and is nearly slipping out to innominate artery. The large arrow indicates the kinking point of the guiding sheath. (E) The guiding sheath (arrowhead) is advanced into the more distal common carotid artery. 


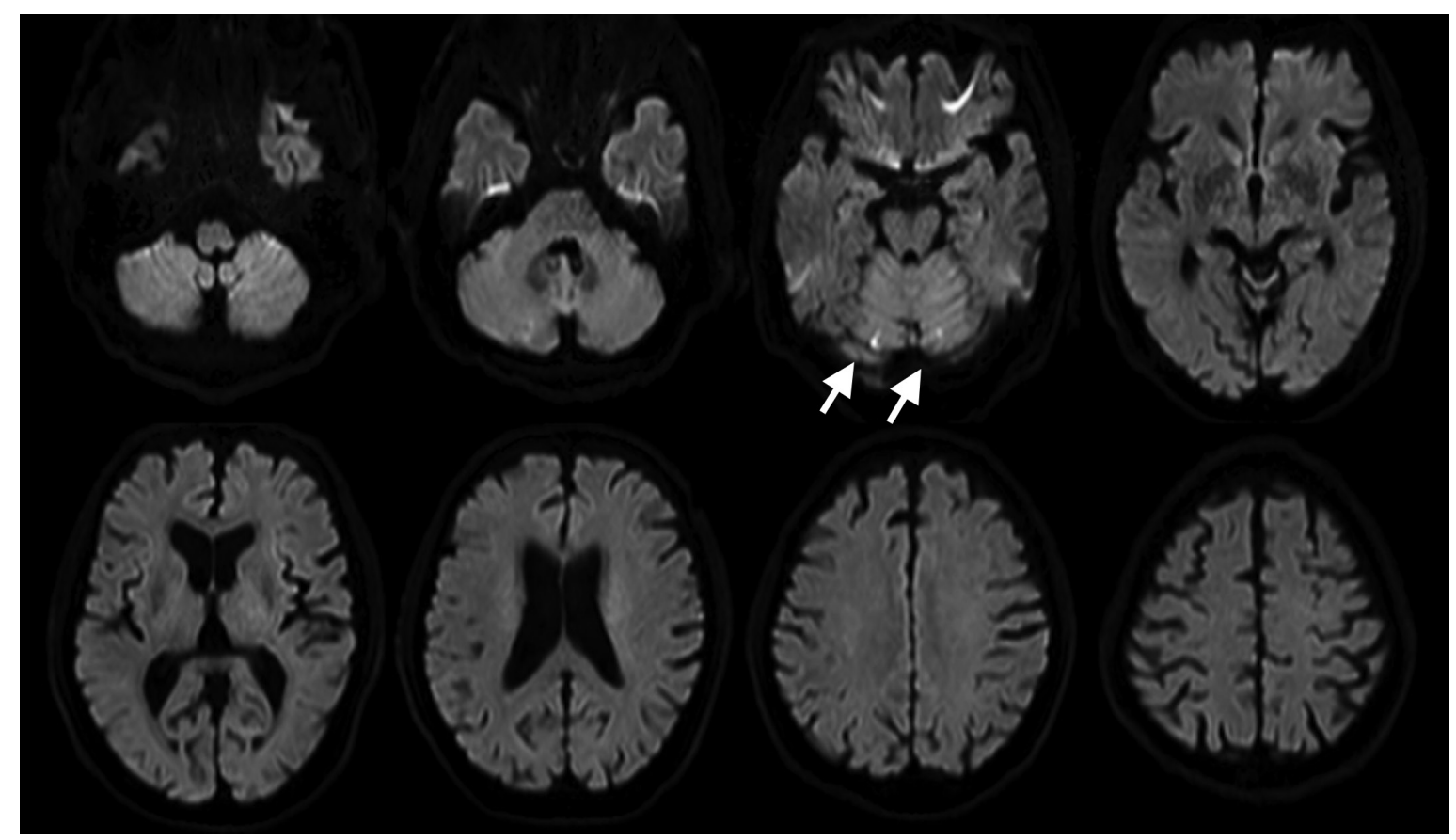

Fig. 6 Postoperative MRI in case 2, showing some scattered high-signal intensities (arrows) in diffusion-weighted images.

subclavian artery to the right common carotid artery comprised a 27-degree hairpin curve (Fig. 4C). Carotid artery stenting (CAS) was selected using a right TRA to avoid intra-aortic-arch manipulation.

Endovascular treatment: Under general anesthesia, a $6 \mathrm{Fr}$ guiding sheath was guided into the subclavian artery using a right TRA. A guidewire was guided into the common carotid artery using a 5 Fr $110-\mathrm{cm}$ pigtail catheter as an inner catheter. The guiding sheath was guided so that it might follow the guidewire, but it fell into the brachiocephalic trunk due to a sharp vascular angle. The guidewire was guided to a more distant area using a pigtail catheter again. At this point, sharp flexion between the subclavian and common carotid arteries made torque transmission to the wire very difficult. Therefore, a torque device (Terumo Corporation) was used to avoid a guidewire migration into internal carotid artery plaques and induce a guidewire into the external carotid artery safely (Fig. 5A). The guidewire was inserted into the maxillary artery, and the pigtail catheter tip was fixed to the origin of the common carotid artery. Then, the guiding sheath was guided into the common carotid artery along the pigtail catheter and the guidewire (Fig. 5B). For the second session of approaching, 19 minutes were required. The sharp vascular angle also influenced the subsequent operations. We tried to perform CAS under distal balloon protection using a Carotid Guardwire PS (Medtronic, Minneapolis, MN, USA). Unfortunately, the 7-cm flexible part of the guiding sheath tip was present at the bifurcation point. When stent was passing the point, the sheath became folded, making stent passage impossible (Fig. 5C and 5D). We had to induce the guiding sheath to a more distant area using a JB2-type inner catheter so that the flexible part is advanced into the common carotid artery, and we finally accomplished CAS (Fig. 5E). The guiding sheath was removed without reversing heparin, and compressive hemostasis with a BLEED SAFE was conducted.

Postoperative course: Vagal reflex-related bradycardia/ hypotension occurred, and the administration of a catecholamine preparation was required for 3 days. MRI revealed a small, infarcted focus in the cerebellum (Fig. 6), but there were no neurologic deficits. Neither radial artery occlusion nor ischemia of the upper limbs was observed, and the patient was discharged, as scheduled.

\section{Discussion}

We presented two patients in whom a 6 Fr guiding sheath was guided into the right carotid artery by avoiding intraaortic-arch manipulation using a pigtail-type inner catheter.

Intra-aortic-arch manipulation may cause cholesterin/ cerebral embolism. ${ }^{1-3)}$ In particular, the risk of these complications is high in shaggy aorta patients, $, 1,5,6)$ and such manipulation should be avoided if other options are available. 
There are some reports regarding usage of a Simmonstype catheter via a TBA. ${ }^{7}$ However, complete avoidance of intra-aortic-arch manipulation is not possible using a Simmons-type catheter. Misaki et al. ${ }^{4}$ successfully avoided intra-aortic-arch manipulation using a pigtail catheter, of which the shape can be maintained even in the brachiocephalic trunk. However, they exchanged catheters twice before guiding a guiding sheath because the pigtail catheter was not long enough to use as an inner catheter; procedures were somewhat complex. We used a pigtail catheter measuring $110 \mathrm{~cm}$ in length, and it was long enough to use as an inner catheter for a $90-\mathrm{cm}$ guiding sheath.

The demerits of catheter exchange via a TBA are as follows: 1) operations with a long wire floating in the air are unstable and difficult; and 2) catheter exchange may trigger spasm of the radial/brachial arteries. ${ }^{8,9)}$ Our method may reduce these risks. However, the size of OptiFlush catheters is limited to 4 to $5 \mathrm{Fr}$; when a smaller system is required, the method proposed by Misaki et al. ${ }^{4)}$ may be useful.

To approach a cervical blood vessel using a conventional catheter via a TRA or TBA, an angle of $\geq 45$ degrees between the subclavian artery and target blood vessel is eligible. ${ }^{10-12)}$ Essentially, pigtail catheters themselves were not designed for usage for peripheral blood vessel, and their flexibility and trackability are less marked than those of standard catheters. On the other hand, advantages and disadvantages are two sides of the same coin. Due to the very firm tip shape which provides a strong supportive power. The induction of a guidewire to a distal area through a $5 \mathrm{Fr}$ pigtail catheter may facilitate subsequent stable operations, even if the angle between the subclavian artery and target blood vessel is $<45$ degrees, as demonstrated in the present cases. However, we have experienced only two cases, and a larger number of cases must be analyzed to evaluate whether our method is more useful than in previous studies. ${ }^{10-12)}$

As indicated in this study, pigtail catheters may be useful in patients with a sharp angle between the subclavian artery and the common carotid artery, but the following limitation is suggested: in case 2, a sharp-angle-mediated approach reduced guidewire operability, and we had to use a torque device to improve the operability. In this study, the use of a torque device improved the operability. However, when the guidewire operation seems to be difficult, the procedure with a pigtail catheter should be considered carefully.

Another limitation of this method is that the pigtail catheter shape reverts soon after removing a guidewire. Hence, after guidewire induction to carotid artery, it is necessary to hold the guidewire until a guiding sheath position becomes stable. If there is no difference in the profile between the sheath and inner catheter, angiography cannot be performed. In this situation, a patient should stay still through the procedure to make no gap between the load map and the actual position of the artery. We performed CAS and coil embolization under general anesthesia, so there was no movement-related gap in the load map. However, in medical institutions where these procedures are performed under local anesthesia, patient's movement must be controlled carefully.

The common limitations of trans-upper-limb approaches are as follows: 1) operability disturbance related to stenosis/ flexion of the right subclavian artery, 2) non-availability for left carotid artery lesions, excluding some variants at the vascular origin, and so forth. When it is necessary to avoid intra-aortic-arch manipulation in such patients, direct puncture of the carotid artery could be an option, or another treatment other than endovascular procedures should be chosen.

\section{Conclusion}

We reported two patients for whom an approach to avoid intra-aortic-arch manipulation was selected, considering its risk. A 6 Fr guiding sheath could be safely and simply guided into the right carotid artery through the right radial artery in the absence of intra-aortic-arch manipulation by adopting a $5 \mathrm{Fr}$ pigtail catheter measuring $110 \mathrm{~cm}$ in length as an inner catheter. Our method is an option to avoid intra-aortic-arch manipulation, facilitating the selection of endovascular treatment for a larger number of patients.

\section{Disclosure Statement}

There is no conflict of interest for the main author and coauthors.

\section{References}

1) Hagiwara N, Toyoda K, Nakayama M, et al: Renal cholesterol embolism in patients with carotid stenosis: a severe and underdiagnosed complication following cerebrovascular procedures. J Neurol Sci 2004; 222: 109-112.

2) Eggebrecht $\mathrm{H}$, Oldenburg $\mathrm{O}$, Dirsch $\mathrm{O}$, et al: Potential embolization by atherosclerotic debris dislodged from 
aortic wall during cardiac catheterization: histological and clinical findings in 7,621 patients. Catheter Cardiovasc Interv 2000; 49: 389-394.

3) Hammer FD, Lacroix V, Duprez T, et al: Cerebral microembolization after protected carotid artery stenting in surgical high-risk patients: results of a 2-year prospective study. J Vasc Surg 2005; 42: 847-853; discussion 853.

4) Misaki K, Uchiyama N, Mohri M, et al: [Guiding-sheath cannulation using a pigtail catheter for transbrachial carotid artery stenting without intra-aortic manipulation]. JNET 2014; 8: 280-284. (in Japanese)

5) Amarenco P, Duyckaerts C, Tzourio C, et al: The prevalence of ulcerated plaques in the aortic arch in patients with stroke. N Engl J Med 1992; 326: 221-225.

6) Meyrier A: Cholesterol crystal embolism: diagnosis and treatment. Kidney Int 2006; 69: 1308-1312.

7) Nanto M, Tsuura M, Takayama $M$, et al: [Carotid artery stenting via a transbrachial artery: techniques and problems]. No Shinkei Geka 2007; 35: 155-160. (in Japanese)
8) Voudris KV, Georgiadou P, Charitakis K, et al: Radial interventions: present and future indications. Curr Treat Options Cardiovasc Med 2016; 18: 2.

9) Marmagkiolis K, Lendel V, Best JF, et al: Management of transradial access complications in the cardiac catheterization lab. Int J Cardiol 2014; 173: 521-524.

10) Iwata $T$, Mori $T$, Miyazaki $Y$, et al: Anatomical features of the vertebral artery for transbrachial direct cannulation of a guiding catheter to perform coil embolization of cerebral aneurysms in the posterior cerebral circulation. Interv Neuroradiol 2015; 21: 381-386.

11) Mizokami $T$, Uwatoko $T$, Inoue $K$, et al: Transbrachial coil embolization for posterior circulation aneurysm in elderly patients using a 4 Fr. Guiding sheath. JNET 2016; 10: 285-290.

12) Montorsi P, Galli S, Ravagnani PM, et al: Carotid artery stenting with proximal embolic protection via a transradial or transbrachial approach: pushing the boundaries of the technique while maintaining safety and efficacy. $J$ Endovasc Ther 2016; 23: 549-560. 